\title{
Torsional oscillations of an auger multifunctional conveyor's screw working body with consideration of the dynamics of a processed medium continuous flow
}

\author{
Oleg Lyashuk ${ }^{1}$, Maria Sokil ${ }^{2}$, Yuriy Vovk ${ }^{1}$, Anna Tson ${ }^{1}$, \\ Andrii Gupka1, Oleksandr Marunych ${ }^{1}$ \\ 1 - Ternopil Ivan Pul'uj National Technical University, Ternopil, Ukraine \\ 2 - Lviv Polytechnic National University, Lviv, Ukraine
}

Keywords:

Screw

Amplitude

Resonance

Conveyor

Article history:

Received

02.05.2018

Received in

revised form

25.09.2018

Accepted

28.09.2018

Corresponding

author:

Yuriy Vovk

E-mail:

vovkyuriy@,

ukr.net

DOI:

$10.24263 / 2304-$

974X-2018-7-3-14

\section{Abstract}

Introduction. The aim of the article is a mathematical model of vibrations of working part of screw conveyer for transporting friable loads taking into account the changes of closeness of the processed environment, speed of circulation of screw, weight while appearing of the phenomenon of resonance.

Materials and methods. It is recesrced the turning vibrations of spiral working part of screw multifunctional conveyer, which appeare as a result of action of processing environment. The recerch was carried out by mathematical modelling method.

Results and discussions. The limiting factors which complicate a design are the unevenness of the division of environment along the length of screw; a body makes flexible vibrations; the motion of the processed environment along the working screw causes the small vibrations of bend, causes appearance of "additional forces". For providing the authenticity of mathematical model ti has been introduced the next suppositions: the working part of screw is turned with the permanent angle speed around its longitudinal which in the undeformed position coincides coincides with an axis OX; the transversal transferring of neutral axis of working screw at the arbitrary moment in perpendicular direction of its undeformed position is determined by the vector (in relation to the fixed frame of reference of OXYZ); the continuous stream of processed environment,while its weight changes on unit length slowly along a screw and moves with permanent relative (in relation to working screw) speed.

A mathematical model takes into account the limitation and supposition for the existent screw conveyer with the length of 2,5 and $3 \mathrm{~m}$, which is used for moving the friable loads with the consistence of $10,20,40 \mathrm{~kg} / \mathrm{m}$. The alternative parameters are the length of the screw,the consistence of the processed environment, the speed of circulation ofthe screw, weight

On the basis of model the dependences of amplitude of vibrations on the lengthof screw, cosistence of the processed environment, the speed of circulation of the screw, weight are built.

Conclusions. A mathematical model allows to improve the process of development and designing of the screw conveyers of the friable loads providing the reliability of process of transportion and system "the processed environment - the screw working part". 


\section{Introduction}

Presently screw conveyers are the part of complex mechanization and automation of manufacturing. Creation of new and improvement of the existent constructions ofscrew conveyers with the extended technological possibilities assists to further development of production and increase of their efficiency of the usige. In the modern constructions of cars the usige of screw mechanisms, the nomenclature of which and the specific of exploitation grow constantly; specific weight of these mechanisms in lift-shifting cars according to the different amounts $40 . .45 \%$ It is established that functional possibilities and technological characteristics of some screw conveyers don't comply the requirements of present time fullyaccording to the signs of expansion of nomenclature of technological processes; results of theoretical and experimental researches, bench equipment etc.. Accordingly the problem of development of the theory and methods of planning of screw conveyers and their descriptions is especially actual has the important value for the economy of the country.

Analysis of the last researches and publications. The conducted analysis of the state of the modern technologies and literary-patent search of constructions of cars and mechanisms for realization of transporting of friable materials [1-3] has showed that in one or another measure they satisfy considerable part of requirements which are pulled out to them, however most constructions of working of working parts of conveyers carry out not only the forward axial moving of material but also implementation of circulating motion, that reduces productivity of such mechanisms. They have a simple construction maximum, differ in acompactness and they are reliable in exploitation. At the same time, the substantial disadvantage ofscrew mechanisms is reduction of their productivity at large frequencies of rotation as a result of increasing of centrifugal forces in the zone of loading that leads to increase of power-hungryness of process of transporting such kinds of devices.

In works [4-8] it was used the method of discrete elements for the analysis of the productivity of screw conveyer including visualization of motion of particles, the angular and axial speed of particles and on the whole twisty moment and total force. It is built the model by application of its periodic border for one step of screw and the particle of friable material, it is shown the dependence of the productivity ofscrew conveyer on operating conditions. It is set that the equability of the stream depends on the construction ofscrew working part and bunker, form of the particles and the force of the friction to walls. The results of this research can present better the usige of screw feeders for specific materials. In-works [9-13] it was conducted the experiment for determination influence of parameterswhich have the most influence on horizontal screw conveyers and also power consumption at transporting of grains.So, in works [14] it is adduced the results of researches saving transporting of agricultural materials on the horizontal and aslope located screw working parts, character of motion of stream of material at its loading [15] and overload ing [16], and also moving material by the vertical screws [17] which it is expedient to take into account during realization of researches of moving of stream of root crops and their cleaning.

Experimental and separate theoretical researches of the dynamics of working parts of screw conveyers [18-19], moving of the processed environment along the last items influence on quantitative and on the other occasion and quality descriptions of their vibrations. Thus, influence of dynamics of environment grows with the increasing of the amount of its relative motion toward the screw working part. At the same time the intensive vibrations of screw working part lead to the considerable increase of dynamic tensions, and as a result to reduction of operating term of work of machine. At certain conditions of exploitation of screw conveyers it can take place both the internal and "external 
"resonances. Envisaging them is possible only on the base of analysis of decisions of mathematical models which are adequate to dynamic process.

At the same time the questions of the influence of the relative motion of processed environment on the turning vibrations of working part which are important for the assement of work of screw conveyer have not found the proper illustration in literature.For their solution of this task there is a mathematical model of vibrations of working part of screw conveyer for transporting friable loads taking into account the change of consistence of the processed environment, the speed of circulation of screw, mass at appearance of thephenomenon of the resonance.

\section{Materials and methods}

\section{Materials}

The object of the research is the working part of multifunctional screw conveyer which is used for transporting of friable materials (forage, chemicals, powders, supplements, etc ). The subject of the research is turning vibrations of screw working part of screw multifunctional conveyer which arise up as a result of action of processed environment.

\section{Description of the device}

The auger compensating multifunctional conveyor is made in the form of a casing 1 with both front 2 and rear 3 supports. Their height can be changed in order to transport materials horizontally and at an angle. The fixed cone trough 4 is firmly fixed to these two supports in several variants - the solid one and with the system of through-holes in its lower part made in a known way. Inside the fixed conical body 4, a conical screw working element 5 with variable steps is installed in the bearing units with the possibility of turning and axial displacement. The bearing bushes 5 are welded to the left 2 and the right 3 supports; in their central openings, the movable cylindrical blocks 6 are set. The bearings are rigidly installed in the middle of blocks; the internal holes of bearing are in contact with the shaft 7 ends of the conical auger 5 with the possibility of axial displacement.

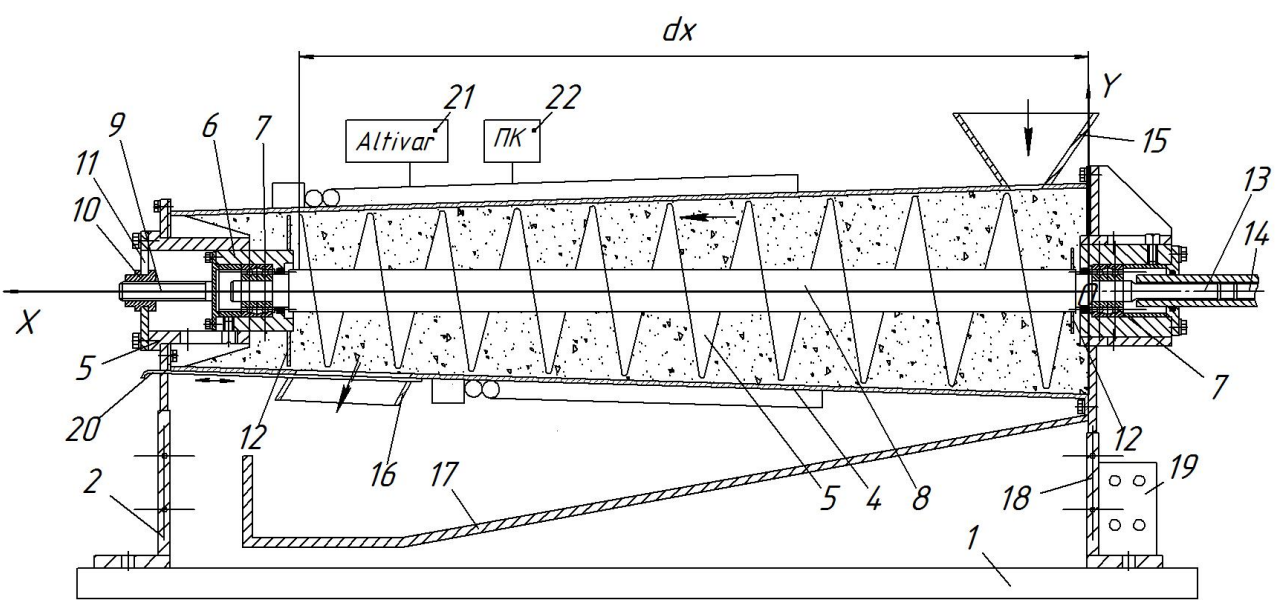

Figure 1. Schematic construction of the auger compensating multifunction conveyor 
The ends of movable blocks 6 are covered with lids. A screw 9 is rigidly welded to the left lid 8 of the cylindrical block 6 . The screw is in contact with the adjusting nut 10 , which is firmly mounted in the axial groove of the lid 11 of the movable cylinder block 6 . In addition, the stopper 13 are firmly fixed to the left and right ends of the shaft 7 of the cylindrical movable block 6 . They prevent the movement of transport mass beyond the screw working body.

Similarly, at the right end of the screw cone working body, the structure is the same, but the difference is that the ends of the shaft 7 are made with an elongated cut 14, to which the drive shaft 15 of the conveyor is connected. In the loading zone, the boot pipe 16 is installed, and in the unloading zone, the discharge nozzle 17 is installed as well. To fix the screw conical working body 5 , in the moving blocks 6 , the axial slots 19 are made with screw fixing elements 20 . The system is controlled from the console 21 .

\section{Results and discussion}

\section{Formulation of the problem}

In case of the screw working body, the task is considerably complicated: firstly, due to the uneven distribution of the medium along the auger length; secondly, the body performs bending oscillations; thirdly, the movement of the processed medium along the working screw, which carries even small bending oscillations, causes the occurrence of 'additional forces', which moments must be factored in the differential equation of torsional oscillations of the system 'processed environment - screw working body'. Thus, in order to study the torsional oscillations of the screw conveyor working body, a 'refined' differential equation of the indicated oscillations should be deduced.

\section{Basic assumptions about the object under study}

a. The working auger screw rotates with a constant angular velocity $\Omega$ around its longitudinal axis, which in the undeformed position coincides with the axis $O X$;

b. The transverse movement of the neutral axis of the working screw at an arbitrary moment in the direction perpendicular to its undeformed position is determined (with respect to the fixed reference frame of the OXYZ) by the vector $\vec{r}=u(x, t) \vec{j}+w(x, t) \vec{k} ;$

c. A continuous flow of the processed medium, which mass per unit length $m$ slowly varies along the length of the screw $(m=m(x))$ and moves with a constant relative (relative to the working auger screw) velocity $V$.

\section{Mathematical model solution}

A differential equation with partial derivatives is known to be a mathematical model of torsional oscillations of a rectilinear body

$$
I \frac{\partial^{2} \theta}{\partial t^{2}}-\frac{\partial}{\partial x}\left(G J \frac{\partial \theta}{\partial x}\right)=Q\left(\theta, \frac{\partial \theta}{\partial t}, \frac{\partial \theta}{\partial x}, \phi\right)
$$

under boundary conditions, which are consistent with the method of body fixation. In Eq. (1) $\theta(x, t)$ - the angle of twisting the elastic body, $I$ - the length moment of inertia relative 
to the axis of rotation, $G$ - the shift module, $J$ - the equatorial moment of the cross section, $Q\left(x, \theta, \frac{\partial \theta}{\partial t}, \frac{\partial \theta}{\partial x}, \phi\right)$ - the nonlinear in conjunction with a set of variables $\theta, \frac{\partial \theta}{\partial t}, \frac{\partial \theta}{\partial x}$ periodic by $\phi=\eta t+\phi_{0}$ function that describes the distribution of external forces moments along the body length relative to the axis of rotation (including the moments of resistance), and $\eta$ - the frequency of periodic perturbation.

In this case, the inertia force [20] of the conditionally selected element of the screw, the working medium of the length $d x$ and its moment relative to the axis of rotation are determined in accordance with the dependencies

$$
\begin{gathered}
\vec{\Phi}_{m .}=2\left(m+m_{1}\right) \Omega\left(\frac{\partial w}{\partial t} \vec{j}-\frac{\partial u}{\partial t} \vec{k}\right) d x \\
M_{m .}^{\Phi} \vec{i}=(u \vec{j}+w \vec{k}) \times \vec{\Phi}_{m .}=-2\left(m+m_{1}\right) \Omega\left(u \frac{\partial u}{\partial t}+w \frac{\partial u}{\partial t}\right) \vec{i} d x,
\end{gathered}
$$

where $m_{1}=m_{1}(x)$ - the mass per unit length of the auger conveyor crew.

Similarly, the inertia force of the processed medium and its moment relative to the axis of rotation is defined, taking into account the medium motion along the deformed screw

$$
\begin{gathered}
\vec{\Phi}_{\text {cep. }}=2 m \Omega V\left(\frac{\partial w}{\partial x} \vec{j}-\frac{\partial u}{\partial x} \vec{k}\right) d x . \\
M_{\text {cep. }}^{\Phi} \vec{i}=(\overrightarrow{u j}+w \vec{k}) \times \vec{\Phi}_{\text {cep. }}=-2 m \Omega V\left(u \frac{\partial u}{\partial x}+w \frac{\partial w}{\partial x}\right) \vec{i} d x .
\end{gathered}
$$

Other inertia forces of the processed medium continuous flow are not considered. The moment of the forces relative to the rotational axis of the working screw is zero and therefore does not affect its torsional oscillations. The above allows presenting a refined differential equation of torsional oscillations of the system 'processed medium continuous flow - screw working body'

$$
\begin{gathered}
\frac{\partial^{2} \theta}{\partial t^{2}}-\frac{G J_{0}}{I_{0}} \frac{\partial^{2} \theta}{\partial x^{2}}=\frac{1}{I_{0}} f\left(x, \theta, \frac{\partial \theta}{\partial t}, \frac{\partial \theta}{\partial x}, \phi\right) \\
\frac{G J_{0}}{I_{0}}=\frac{G}{\rho}
\end{gathered}
$$

where:

$$
f\left(x, \theta, \frac{\partial \theta}{\partial t}, \frac{\partial \theta}{\partial x}, \phi\right)=Q\left(x, \theta, \frac{\partial \theta}{\partial t}, \frac{\partial \theta}{\partial x}, \phi\right)-2 \Omega\left[\left(m+m_{1}\right)\left(u \frac{\partial u}{\partial t}+w \frac{\partial w}{\partial t}\right)+m V\left(u \frac{\partial u}{\partial x}+w \frac{\partial w}{\partial x}\right)\right]
$$

$G$ - the shift module, $I_{0}$ - the length moment of the inertia relative to the axis of a working screw together with the processed medium, $J_{0}$ - the equatorial moment of the working screw cross-section and the values below are considered constant.

Further calculations relate to the development and study of the differential equation solution (6) under boundary conditions 


$$
\theta(x, t)_{\mid x=0}=\theta(x, t)_{\mid x=l}=0
$$

which correspond to the twisting oscillations of the auger working body with free ends, and $l-$ the distance between the bearings, in which it rotates. Based on the following physically grounded statements, the boundary problem Eq. (6), Eq. (7) can be solved:

1. The maximum value of the right-hand side of differential equation Eq. (6) is a small value in comparison with the maximum value of the 'restoring moment of torsion', that is, the second summand of its left-hand side;

2. Functions of the deflection $u(x, t), w(x, t)$ of the screw working body are determined [21] by a wave form with an amplitude $a$, a wave number $\lambda_{k}=\frac{k \pi}{l}$, a frequency $\omega_{k}=\sqrt{\left(\frac{k \pi}{l}\right)^{4} \frac{E \bar{I}_{0}}{m+m_{1}}-\Omega^{2}}, \bar{I}_{0}$ - the inertia moment of the working screw crosssection relative to the neutral axis of the cross-section, which is perpendicular to the plane of oscillation. In this case, the right-hand side of differential equation Eq. (6) can be presented in the form

$$
f(x, \theta, \ldots, \phi)=Q(x, \theta, \ldots, \phi)-4 \Omega a^{2}\left(2\left(m+m_{1}\right) \omega_{k} \sin ^{2} \lambda_{k} x \sin 2 \psi_{k}+m V \lambda_{k} \sin 2 \lambda_{k} x \cos 2 \psi_{k}\right), \psi_{k}=\omega_{k} t+\psi_{0}
$$

where $\psi_{0}$ - a constant. Thus, the problem solution was reduced to developing a solution of differential Eq. (6), considering Eq. (8) under boundary conditions Eq. (7).

The most effective methods of studying 'weakly nonlinear' oscillatory systems with lumped masses or distributed parameters are methods based on the main idea of perturbation methods [22-25]. In relation to the considered problem, the application of the principle of one-frequency oscillations and the basic idea of the Bubnov Galerkin method [21, 29-30] allow reducing the boundary problem Eq. (6), Eq. (7) to integration of the system of ordinary quasilinear differential equations

$$
\begin{aligned}
& \ddot{T}_{1}(t)+\left(\frac{\pi}{l}\right)^{2} \frac{G J_{0}}{I_{0}} T_{1}=\frac{1}{l I_{0}}\left\{\int_{0}^{l} Q\left(x, T_{1} \sin \frac{\pi}{l} x, \ldots, \phi\right) \sin \frac{\pi}{l} x d x-\frac{16 l}{3 \sqrt{\pi}} \Omega a^{2}\left(m+m_{1}\right) \omega_{1} \sin 2 \psi\right. \\
& T_{2}(t)+\left(\frac{2 \pi}{l}\right)^{2} \frac{G J_{0}}{I_{0}} T_{2}=\frac{1}{l I_{0}}\left\{\int_{0}^{l} Q\left(x, T_{2} \sin \frac{2 \pi}{l} x, \ldots, \phi\right) \sin \frac{2 \pi}{l} x d x-4 \Omega a^{2} l m V \cos 2 \psi\right.
\end{aligned}
$$

Based on dependences Eq. (9), Eq. (10), the most interesting cases of torsional oscillations from the theoretical and practical point of view are cases:

$$
\begin{gathered}
\text { a. } \frac{\pi}{l} \sqrt{\frac{G J_{0}}{I_{0}}} \approx \sqrt{\left(\frac{2 \pi}{l}\right)^{4} \frac{E \bar{I}_{0}}{m+m_{1}}-\Omega^{2}}, \quad \text { b. } \frac{2 \pi}{l} \sqrt{\frac{G J_{0}}{I_{0}}} \approx \sqrt{\left(\frac{2 \pi}{l}\right)^{4} \frac{E \bar{I}_{0}}{m+m_{1}}-\Omega^{2}}, \\
\text { c. } \frac{k \pi}{l} \sqrt{\frac{G J_{0}}{I_{0}}} \approx \eta .
\end{gathered}
$$


These cases are called resonant. The case (c) is defined by external factors, then, the cases (a) and (b) - by bending oscillations of the working screw. In addition, the case (a) considers purely bending oscillations and rotations of the working screw, then, the case (b) - the processed medium additional motion along the working screw, more precisely, the moment relative to the rotation axis of the Coriolis force of the inertia of the processed medium.

\section{Mathematical model discussion}

According to individual studies [26-28], combination resonances of higher orders are less important than the above-mentioned ones;

The methods of studying the resonance phenomena on different frequencies do not fundamentally differ. Therefore, the general statements based on the generalization of the main idea of the Van der Pol method to the differential equation Eq. (9) or Eq. (10) are presented.

Thus, the resonance oscillations under the conditions a), b), c) are described by the dependence $T_{i}(t)=\bar{a}_{i}(t) \cos \left(\bar{\omega}_{i} t+\vartheta_{i}(t)\right)$, where $\bar{\omega}_{i}$ is one of the frequencies, on which resonance oscillations are considered, and the parameters $\bar{a}_{i}(t)$ and $\vartheta_{i}(t)$ (the amplitude and phase difference of resonant oscillations) are determined by the differential equations:

a. For the resonance at the first angular rotation velocity of the screw working body

$$
\begin{gathered}
\frac{d a_{1}}{d t}=\frac{1}{2 \pi^{2} \bar{\omega}_{1} l I_{0}} \int_{0}^{l} \int_{0}^{2 \pi} \int_{0}^{2 \pi} Q\left(x, \bar{a}_{1} \sin \frac{\pi}{l} x \cos \psi, \ldots, \phi\right) \sin \frac{\pi}{l} x \sin \varphi d x d \varphi d \phi- \\
-\frac{16 l}{3 \bar{\omega}_{1} I_{0}} \Omega_{1} a^{2}\left(m+m_{1}\right) \omega_{1} \cos \gamma_{1}, \\
\frac{d \gamma_{1}}{d t}=\frac{\pi}{l} \sqrt{\frac{G J_{0}}{I_{0}}}-\sqrt{\left(\frac{2 \pi}{l}\right)^{4} \frac{E \bar{I}_{0}}{m+m_{1}}-\Omega_{1}^{2}}+ \\
+\frac{1}{2 \pi^{2} \bar{\omega}_{1} \bar{a}_{1} l I_{0}} \int_{0}^{l} \int_{0}^{2 \pi} \int_{0}^{2 \pi} Q\left(x, \bar{a}_{2} \sin \frac{\pi}{l} x \cos \psi, \ldots, \phi\right) \sin \frac{\pi}{l} x \cos \varphi d x d \varphi d \phi+\frac{16 l}{3 \bar{\omega}_{1} \bar{a}_{1} I_{0}} \Omega_{1} a^{2}\left(m+m_{1}\right) \omega_{1} \sin \gamma_{1} ;
\end{gathered}
$$

b. For the resonance at the second angular rotation velocity of the screw working body

$$
\begin{gathered}
\frac{d a_{2}}{d t}=\frac{1}{2 \pi^{2} \bar{\omega}_{2} l I_{0}} \int_{0}^{l} \int_{0}^{2 \pi} \int_{0}^{2 \pi} Q\left(x, a_{1} \sin \frac{2 \pi}{l} x \cos \psi, \ldots, \phi\right) \sin \frac{2 \pi}{l} x \sin \varphi d x d \varphi d \phi+ \\
+\frac{2 \Omega_{2} a^{2} V l m \pi}{\bar{\omega}_{2} I_{0}} \sin \gamma_{2}, \\
\frac{d \gamma_{2}}{d t}=\frac{2 \pi}{l} \sqrt{\frac{G J_{0}}{I_{0}}}-\sqrt{\left(\frac{2 \pi}{l}\right)^{4} \frac{E \bar{I}_{0}}{m+m_{1}}-\Omega_{2}^{2}}+ \\
+\frac{1}{2 \pi^{2} \bar{\omega}_{2} \bar{a}_{2} l I_{0}} \int_{0}^{l} \int_{0}^{2 \pi} \int_{0}^{2 \pi} Q\left(x, a_{2} \sin \frac{\pi}{l} x \cos \psi, \ldots, \phi\right) \sin \frac{\pi}{l} x \cos \varphi d x d \varphi d \phi-\frac{2 \Omega_{2} a^{2} V l m \pi}{\bar{\omega}_{2} \bar{a}_{2} I_{0}} \cos \gamma_{2} ;
\end{gathered}
$$


c. For the resonance on the frequency of the external periodic perturbation

$$
\begin{aligned}
& \frac{d \bar{a}_{3}}{d t}=\frac{1}{2 \pi l \mu I_{0}} \int_{0}^{l} \int_{0}^{2 \pi} Q\left(x, \bar{a}_{3} \sin \frac{\pi}{l} x \cos \left(\gamma_{3}+\varphi\right), \ldots, \varphi\right) \sin \left(\gamma_{3}+\varphi\right) \sin \frac{\pi}{l} x d x d \varphi \\
& \frac{d \gamma_{3}}{d t}=\frac{\pi}{l} \sqrt{\frac{G J_{0}}{I_{0}}}-\mu+\frac{1}{2 \pi l \bar{\omega}_{1} I_{0} \bar{a}_{3}} \int_{0}^{l} \int_{0}^{2 \pi} Q\left(x, \bar{a}_{3} \sin \frac{\pi}{l} x \cos \left(\gamma_{3}+\varphi\right), \ldots, \varphi\right) \cos \left(\gamma_{3}+\varphi\right) \sin \frac{\pi}{l} x d x d \varphi \\
& \text { where } \bar{\omega}_{1}=\frac{\pi}{l} \sqrt{\frac{G J_{0}}{I_{0}}}, \bar{\omega}_{2}=\frac{2 \pi}{l} \sqrt{\frac{G J_{0}}{I_{0}}} .
\end{aligned}
$$

Consequently, the internal resonances occur only due to a certain correlation between the frequency of the own transverse and torsional oscillations, more precisely, due to the distribution of masses in the system 'processed medium - auger working body', its geometrical dimensions and the angular velocity of the working screw. According to equations Eq. (11) and Eq. (12), in Figure 1 and Figure 2, under the condition $Q=Q\left(x, \theta, \frac{\partial \theta}{\partial x}, \frac{\partial \theta}{\partial t}, \phi\right)=\delta\left(\frac{\partial \theta}{\partial t}\right)^{s}(\delta, s-$ steels $)$, the amplitudes of torsional oscillations of the working screw during the transition through the resonance are presented for different lengths of the working screw, and different values of its densities (see figure 1), and different values of masses per unit length of the processed medium and its velocity (see Figure 2).

On the basis of theoretical data it is shown the results of experimental researches of dependence of the productivity of the inclined іскуц conveyer from the internal diameter of casing of $D$, epy angle of slope of conveyer $\gamma$ and frequency of rotation of working part $n$ during the transporting of friable material (wheats). It is deduced the equation of regression for determination the productivity of transportion of wheat.

During transportion of wheat :

$$
Q_{(D, \gamma, n)}=3,387-117,52 D+2,49 \cdot 10^{-2} \gamma-8,92 \cdot 10^{-3} n-0,328 D \gamma++0,234 D n-3,4 \cdot 10^{-5} \gamma n+880 D^{2}
$$

On the basis ofthe experemental researches it was set the maximal value of the productivity $-6,4 \mathrm{t} / \mathrm{hour}$, and minimum $-0,2 \mathrm{t} / \mathrm{hour}$. The basic factors which influence on increasing the productivity are the external diameter of screw working part and amount of turns and at the increase of angle of slope of conveyer the productivity is reducing. 


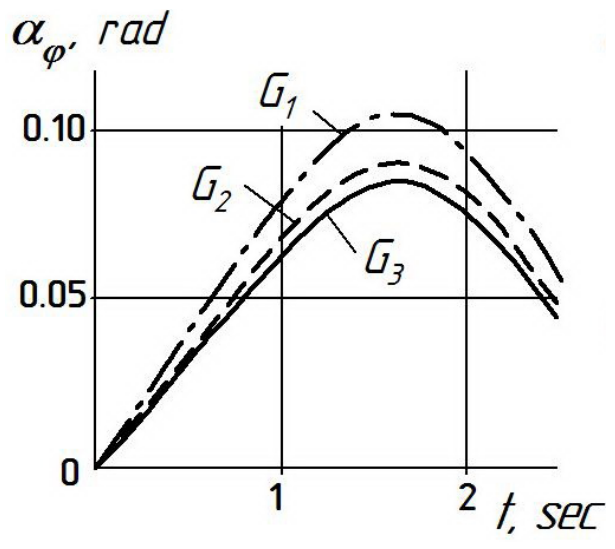

$a$

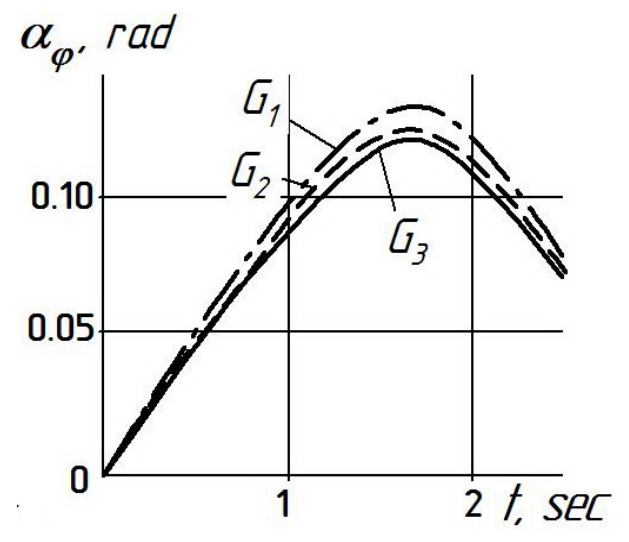

b

$$
\begin{gathered}
m=m_{1}=10 \mathrm{~kg} / \mathrm{m}, E=2 \cdot 10^{11} \mathrm{MN} / \mathrm{m}^{2}, \\
\bar{I}_{0}=2.78 \cdot 10^{-7} \mathrm{~m}^{4}, G_{1}=1.6 \cdot 10^{11} \mathrm{MN} / \mathrm{m}^{2}, \\
G_{2}=1.4 \cdot 10^{11} \mathrm{MN} / \mathrm{m}^{2}, G_{3}=1.3 \cdot 10^{11} \\
\mathrm{MN} / \mathrm{m}^{2}, l=2.5 \mathrm{~m}
\end{gathered}
$$

Figure 2. Change in the amplitude of torsional oscillations of the working screw during the transition through the resonance on the first frequency of transverse oscillations under the following values of parameters

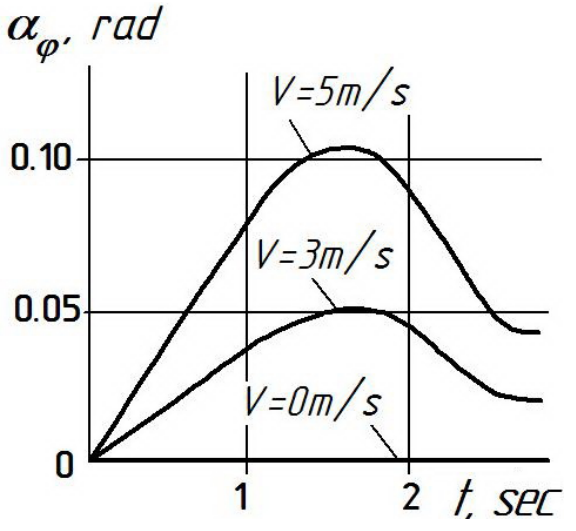

a

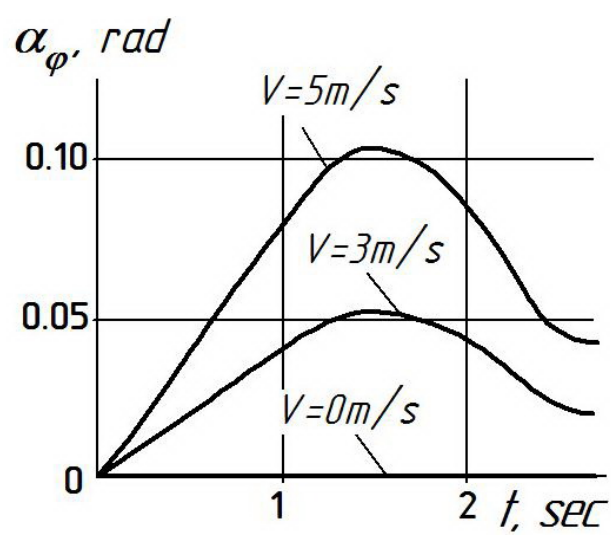

b

$m=40 \mathrm{~kg} / \mathrm{m}, \quad m_{1}=10 \mathrm{~kg} / \mathrm{m}, \quad E=2 \cdot 10^{11} \quad m=20 \mathrm{~kg} / \mathrm{m}, \quad m_{1}=10 \mathrm{~kg} / \mathrm{m}, \quad E=2 \cdot 10^{11}$ $\mathrm{MN} / \mathrm{m}^{2}, \bar{I}_{0}=2.78 \cdot 10^{-7} \mathrm{~m}^{4}, G_{1}=1.3 \cdot 10^{11} \mathrm{MN} / \mathrm{m}^{2}, \quad \bar{I}_{0}=2.78 \cdot 10^{-7} \mathrm{~m}^{4}, \quad G_{1}=1.3 \cdot 10^{11}$ $\mathrm{MN} / m^{2}, l=3 m$ $\mathrm{MN} / m^{2}, l=3 m$

Figure 3. Change in the amplitude of torsional oscillations of the working screw during the transition through the resonance on the second frequency of transverse oscillations under the following values of parameters 


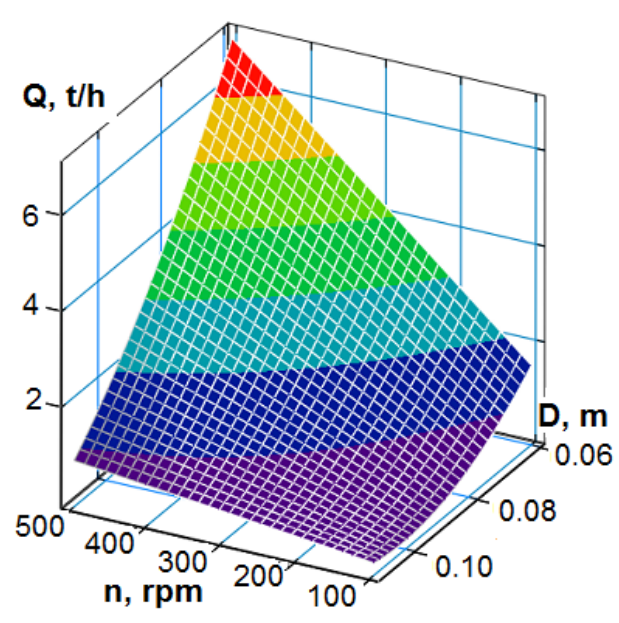

$\boldsymbol{a}$

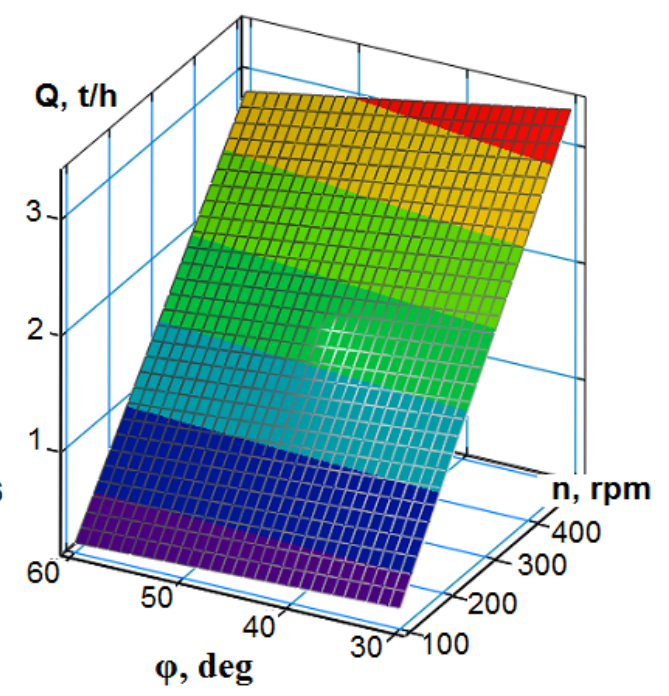

$b$

Figure 4 . Surface of review of the change of the productivity of conveyer $Q$ during transportion as a functional:

$$
\mathbf{a}-Q=f_{(Q)}(D ; n) ; \quad \mathbf{b}-Q=f_{(Q)}(n ; \gamma)
$$

\section{Conclusions}

Based on the developed method of studying the torsional oscillations of the screw working auger conveyor with consideration of its transverse oscillations and the motion of the processed medium continuous flow, the following conclusions are made:

1. The constant angular rotation velocity of the screw working body decelerates the frequency of the own bending oscillations of the screw working body;

2. Relative movement of the processed medium along the screw working body, which rotates and performs transverse oscillations, acts additionally on the body as the periodic torque;

3. According to the above-mentioned, the resonances can occur during the torsional oscillations of the screw working body on the first fundamental and the second frequencies of the twisting oscillations of the working body and on the frequency of external periodic perturbation;

4. The amplitude of the transition through the resonance on the fundamental frequency of bending oscillations assumes smaller value for screw working bodies with greater torsional rigidity;

5. The amplitude of torsional oscillations during the transition through the resonance on the second fundamental frequency is higher due to the larger values of the quantity of processed medium relative motion; 
6. The amplitude of torsional oscillations during the 'fast' transition through the resonance on the frequency of external or internal perturbations is smaller than during the 'slow' one.

The obtained results can serve as a basis for choosing the basic technological and operational parameters of a screw multifunctional conveyor. The results reliability is confirmed by obtaining in the limiting case under $V \rightarrow 0$ the known [3], which relate to the influence of bending oscillations of the elastic screw on the torsional ones, without considering the motion of the processed medium.

The results of modeling correlate (call) with the results of researches [1, 8, 15, 21, 29]. The received results should be followed for further planning the similar screw conveyers with similar changeable parameters.

\section{References}

1. Hu G., Chen J., Jian B., Wan H., Liu L., (2010), Modeling and simulation of transportation system of screw conveyors by the discrete element method. International Conference on Mechanic Automation and Control Engineering, MACE 2010, Article number 5536244, pp. 927-930.

2. Zareiforoush H., Komarizadeh M.H., Alizadeh M.R., (2010), Effect of crop-screw parameters on rough rice grain damage in handling with a horizontal screw conveyor. Journal of Food, Agriculture and Environment, 8(3-4), pp. 494-499.

3. Fernandez J. W., Cleary P.W., McBride W., (2009), Effekt of screw desing on hopper draw dawn by a horizontal screw feeder, Seventh International Conference on CFD in the Minerals and Process Industries CSIRO, Melbourne, Australia 9-11 Decem, pp. 1-6.

4. Zareiforoush H., Komarizadeh M.H., Alizadeh M.R., (2010), Screw conveyors power and throughput analysis during horizontal handling of paddy grains, Agricultural Science, 2(2), pp. $147-157$.

5. Roberts A.W., Bulk Solids, (2015), Optimizing Screw Conveyors, Chemical engineering, 122(2), pp. 62-67.

6. Yao Y.P., Kou Z.M., Meng W.J., Han G., (2014), Overall Performance Evaluation of Tubular Scraper Conveyors Using a TOPSIS-Based Multiattribute Decision-Making Method, The Scientific World Journal.

7. Owen P.J., Cleary P.W., (2010), Screw conveyor performance: comparison of discrete element modelling with laboratory experiments, Progress in computational fluid dynamics, 10(5-6), pp. 327-333.

8. Manjula E. V. P. J., Ariyaratne W. H., Ratnayake, C., \& Melaaen, M. C., (2017), A review of CFD modelling studies on pneumatic conveying and challenges in modelling offshore drill cuttings transport, Powder Technology, 305, pp. 782-793.

9. Tripathi N., Sharma A., Mallick S. S., Wypych P. W., (2015), Energy loss at bends in the pneumatic conveying of fly ash. Particuology, 21, pp. 65-73.

10. Xiaoxia S., Wenjun M., Yuan Y., (2017), Design method of a vertical screw conveyor based on Taylor-Couette-Poiseuille stable helical vortex. Advances in Mechanical Engineering, 9(7), 1687814017714984.

11. Tian Y., Yuan P., Yang F., Gu J., Chen M., Tang J., ... Cheng Q., (2018), Research on the Principle of a New Flexible Screw Conveyor and its Power Consumption. Applied Sciences, 8(7), pp. 1038.

12. Chen L. Q., Wang B., \& Ding H., (2009), Nonlinear parametric vibration of axially moving beams: asymptotic analysis and differential quadrature verification, In Journal of Physics: Conference Series, 181(1), pp. 1-8. 
13. Hevko R.B., Synii S.V., Pankiv M.R., Varholiak M.A., (2014), Development and Analysis of Machine Operation for Energy Saving Technologies of Root Crop Harvesting, Bulletin of Engineering Academy of Ukraine, 4, pp. 46-52.

14. Lyashuk O.L., Rogatynska O.R., Serilko D.L., (2015), Modelling of the vertical screw conveyer loading, INMATEH: Agricultural Engineering, 45, pp. 87-94.

15. Hevko R.B., Tkachenko I.H., Synii S.V., Flonts I.V., (2016), Development of design and investigation of operation processes of small-sclale root crop and potato harvesters. INMATEH: Agricultural engineering, 49, pp. 53-60.

16. Lyashuk O.L., Sokil M.B., Dovbush A.P., (2016), Dynamics of flexible elements of drive systems with variable contact point to the pulleys. INMATEH: Agricultural Engineering, 48, pp. 119-124.

17. Hevko I., Lobachivskyi R., Diachun A., (2011), Experimental investigations of mixing the bulk materials with a mixer by pouring them into another container, Interuniversity Collection ("Engineering Mechanics") "Scientific Notes" LNTU, 5, pp. 51-55.

18. Lyashuk O., Pyndus T., Marunych O., Sokil M., (2016), Longitudinal-angular oscillation of wheeled vehicles with non-linear power characteristics of absorber system, Scientific Journal of the Ternopil National Technical University, 83(2), pp. 82-89.

19. Pavlovskiy M. A., Putiata T. V., (1985), Theoretical mechanics, Kyiv, Ukraine.

20. Rohatynskyi R. M., (2014), Scientific and Applied Fundamentals for Creation of Screw Transport and Technological Mechanisms, Ternopil, Ukraine: Publishing house of TNTU.

21. Mitropolskiy Yu. A., Moseyenkov B. I., (1976), Asymptotic solutions of partial differential equations, Kyiv, Ukraine.

22. Stotsko Z., Sokil B., Topilnytskyj V., (2000), Das Unlinear parametrischmodell der Dreimassenmaschienen fur die Vibrationsvolumenbehandlung und ihre Streuladung, Kwartalnik Naukowo-Techniczny Maszyny Dzwigowo-Transportowe, 3, pp. 50-62.

23. Stotsko Z.A., Sokil B., Topilnytskyj V., (2007), Complex mathematical model and optimization of vibration volumetric treatment for surfaces of machine parts, J. of Achievements in Materials and Manufacturing Engineering, 24, pp. 283-290.

24. Hevko B.M., Pavel'chuk Yu.F., (2017), Modeling the fluctuations of the mechanical system of a suspension hole: theoretical analysis, Innovative solutions in Modern Science, 10(1), pp. 1-9.

25. Geruk S.M., Dovbysh A.P., (2016), Influence of vibration separator oscillations perturbations on the grain mixture dynamics, Construction, production and operation of agricultural machinery, 46, pp. 124-132.

26. Kuzio I.V., Kharchenko Ye. V., Sokil M. B., (2007), Dynamic processes in environments characterized by longitudinal motion and the effect of boundary conditions on the amplitude and frequency of their oscillations, Vibration in techniques and technology, 48(3), pp. 53-56.

27. Sokil M.B., Khytriak O.I., (2011), Wave theory of motion in the study of fluctuations of flexible drive elements and transportation factoring in their longitudinal motion, Military Technical Collection, 1, pp. 102-105.

28. Hevko, B., Lyashuk, O., Sokil, M., Skyba, O., Marunych, O., Shmatko, D., (2018), Dynamics of auger working body of a multifunctional conveyor. Bulletin of the Karaganda University. «Mathematics» series, 89(1), pp. 105-113.

29. Lyashuk O.L., Sokil M.B., Klendiy V.M., Skyba O.P., Slobodian L.M., (2018), Mathematical model of bending vibrations of a horizontal feeder-mixer along the flow of grain mixture, INMATEH: Agricultural engineering, 55(2), pp. 33-42. 